\title{
Effects of Chemical Potential on Hadron Masses in the Phase Transition Region *
}

QCD-TARO Collaboration: Ph. de Forcrand ${ }^{a}$, M. García Pérez ${ }^{b}$, T. Hashimoto ${ }^{c}$, S. Hioki $^{d}$, Y. Liu $^{e}$ H. Matsufuru ${ }^{j}$, O. Miyamura ${ }^{e}$, A. Nakamura ${ }^{g}$, I.-O. Stamatescu ${ }^{f, h}$, T. Takaishi ${ }^{i}$ and T. Umeda ${ }^{e}$

${ }^{a}$ ETH-Zürich, CH-8092 Zürich, Switzerland

${ }^{b}$ Dept. Física Teórica, Universidad Autónoma de Madrid, E-28049 Madrid, Spain

${ }^{c}$ Dept. of Appl. Phys., Fac. of Engineering, Fukui Univ., Fukui 910-8507, Japan

${ }^{d}$ Dept. of Physics, Tezukayama Univ., Nara 631-8501, Japan

${ }^{e}$ Dept. of Physics, Hiroshima Univ., Higashi-Hiroshima 739-8526, Japan

${ }^{f}$ Inst. Theor. Physik, Univ. of Heidelberg, D-69120 Heidelberg, Germany

${ }^{g}$ Res. Inst. for Inform. Sci. and Education, Hiroshima Univ., Higashi-Hiroshima 739-8521, Japan

${ }^{h}$ FEST, Schmeilweg 5, D-69118 Heidelberg, Germany

${ }^{i}$ Hiroshima University of Economics, Hiroshima 731-0192, Japan

${ }^{j}$ RCNP, Osaka University, Osaka 567, Japan

We study the response of hadron masses with respect to chemical potential at $\mu=0$. Our preliminary results of the pion channel show that $\partial m / \partial \mu$ in the confinement phase is significantly larger than that in the deconfinement phase, which is consistent with the chiral restoration.

\section{Introduction}

As suggested by QCD sum rule analysis [1], hadron masses may be affected by density effects. This may explain some results of heavy ion collision experiments such as dilepton spectra and $\mathrm{J} / \Psi$ suppression.

It is difficult to introduce density effects in lattice QCD calculations due to the well-known "complex action" problem. Here we calculate the response of hadron masses to chemical potential, $\partial m / \partial \mu$, on dynamical configurations with $\mu=0$. Since simulations are done at $\mu=0$, there is no difficulty in obtaining $\partial m / \partial \mu$. We investigate the dependence of $\partial m / \partial \mu$ with the temperature.

\section{Formulation}

We use 2 flavors of staggered quarks. The effective action to simulate $N_{f}$ fermion flavors is

$S_{\text {eff }}=S_{G}+S_{F}$

$\overline{{ }^{*} \text { Talk presented by T.Takaishi }}$

where $S_{G}$ is the standard plaquette action and

$S_{F}=\frac{N_{f}}{4} \operatorname{Tr} \ln M(U, \mu)$

where $M(U, \mu)$ is the staggered fermion Matrix.

The zero momentum hadron correlation function $G(t)$ is given by

$G(t)=\sum_{x}<H(x, t) H(0,0)^{\dagger}>$

and

$$
\begin{aligned}
& <H(x, t) H(0,0)^{\dagger}> \\
& =\int d U H(x, t) H(0,0)^{\dagger} \exp \left(-S_{e f f}\right) / Z
\end{aligned}
$$

where $Z$ is the partition function.

Taking a derivative of the hadronic correlator with respect to $\mu$.

$$
\begin{aligned}
& \frac{\partial<H(x, t) H(0,0)^{\dagger}>}{\partial \mu}=<\frac{\partial C(x, t)}{\partial \mu}>(5) \\
& -<C(x, t) \frac{\partial S_{F}}{\partial \mu}>+<C(x, t)><\frac{\partial S_{F}}{\partial \mu}>
\end{aligned}
$$


where $C(x, t)=H(x, t) H(0,0)^{\dagger}$. We calculate eq. (5) on dynamical configurations with $\mu=0$. In the case of $\mu=0$ eq. (5) can be simplified using the following facts:

(A) $\partial S_{F} / \partial \mu$ corresponds to the fermion number operator. Thus, the average of the fermion number operator at $\mu=0$ is zero: $\left\langle\frac{\partial S_{F}}{\partial \mu}>=0\right.$.

(B) On each configuration the value of $\partial S_{F} / \partial \mu$ is purely imaginary [2]. Thus, the value of $<C(x, t) \frac{\partial S_{F}}{\partial \mu}>$ is also purely imaginary provided that the operator $C(x, t)$ is real. This is indeed the case if we consider $C(x, t)$ for mesons made up of degenerate quarks.

Using the facts (A) and (B) above we derive

$\frac{\partial<H(x, t) H(0,0)^{\dagger}>}{\partial \mu}=<\frac{\partial C(x, t)}{\partial \mu}>$

for mesons consisting of degenerate quarks.

In the spectral representation,

$G(t)=\sum_{i} A_{i} \cosh \left(m_{i}\left(t-N_{t} / 2\right)\right)$.

Taking a derivative of eq. (7) with respect to $\mu$ we obtain

$$
\begin{aligned}
& \frac{\partial G(t)}{\partial \mu}=\sum_{i}\left[\frac{\partial A_{i}}{\partial \mu} \cosh \left(m_{i}\left(t-N_{t} / 2\right)\right)\right. \\
& \left.+\frac{\partial m_{i}}{\partial \mu} A_{i}\left(t-N_{t} / 2\right) \sinh \left(m_{i}\left(t-N_{t} / 2\right)\right)\right] .
\end{aligned}
$$

Our procedure to obtain $\partial m / \partial \mu$ is as follows. First we determine $A_{i}$ and $m_{i}$ by fitting correlation function data to eq. (7). Substituting the values of $A_{i}$ and $m_{i}$ into eq. (8) we fit the data of $\frac{\partial G(t)}{\partial \mu}$ to eq. (8). Then we obtain $\partial m_{i} / \partial \mu$ and $\partial A_{i} / \partial \mu$ as fitting parameters.

\section{Definition of $\partial / \partial \mu$}

We study the two flavor case ( $u$ and $d$ quarks). In this case, we have two independent chemical potentials, $\mu_{u}$ and $\mu_{d}$. Instead, the following combinations are convenient, $\mu_{S}=\left(\mu_{u}+\mu_{d}\right) / 2$ and $\mu_{V}=\left(\mu_{u}-\mu_{d}\right) / 2$ whith $\mu_{S}$ the usual chemical potential corresponding to baryon number. Then derivatives with respect to $\mu_{S}$ and $\mu_{V}$ are

$\frac{\partial}{\partial \mu_{S}}=\frac{\partial}{\partial \mu_{u}}+\frac{\partial}{\partial \mu_{d}}=\frac{\partial}{\partial \mu_{u}}-\frac{\partial}{\partial \mu_{\bar{d}}}$ $\frac{\partial}{\partial \mu_{V}}=\frac{\partial}{\partial \mu_{u}}-\frac{\partial}{\partial \mu_{d}}=\frac{\partial}{\partial \mu_{u}}+\frac{\partial}{\partial \mu_{\bar{d}}}$.

For degenerate systems of $u$ and $d$ quarks,

$\frac{\partial C_{u \bar{d}}}{\partial \mu_{S}}=\frac{\partial C_{u \bar{d}}}{\partial \mu_{u}}-\frac{\partial C_{u \bar{d}}}{\partial \mu_{\bar{d}}}=0$.

at $\mu_{u}=\mu_{d}=0$. In this study we analize $\partial / \partial \mu_{V}$ which gives non-trivial results even with degenerate quarks. In the following $\partial / \partial \mu$ stands for $\partial / \partial \mu_{V}$.

$\beta=5.20 \quad m=0.025$

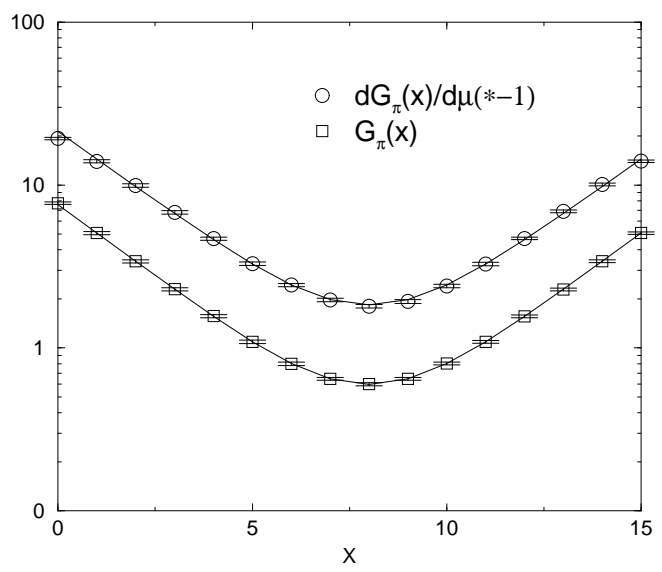

Figure 1. The pion correlation function, $G_{\pi}(x)$ and its derivative with respect to the chemical potential, $\frac{\partial G_{\pi}(x)}{\partial \mu}$ at $\beta=5.20$. $\frac{\partial G_{\pi}(x)}{\partial \mu}$ gives negative values. To plot them in logarithmic scale, they are multiplied by -1 . Single pole fitting results are also shown, represented by solid lines.

\section{Preliminary results}

We present preliminary results of $\partial m / \partial \mu$ for $N_{f}=2$ staggered quarks. Simulations are done on a lattice of size $16 \times 8 \times 8 \times 4$ at $m_{q}=0.025$ with $\beta=5.20,5.26,5.32$ and 5.34. We use the $\mathrm{R}$-algorithm to generate configurations. The finite temperature transition occurs at $\beta \approx 5.28$ 


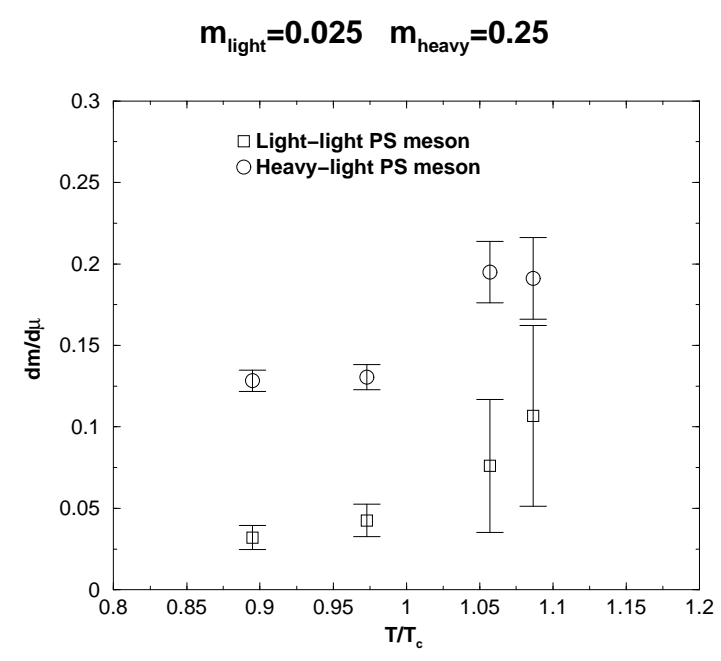

Figure 2. $\partial m / \partial \mu$ of light-light and heavy-light pseudoscalar mesons as a function of $T / T_{c}$.

[3 and the above $\beta$ values are translated to $T / T_{c}=0.90,0.97,1.06$ and 1.09 respectively.

We measure the pion screening mass. The quark propagator is calculated with $m_{q}=0.025$ (light) and 0.25 (heavy). Then we construct the pion correlator with light-light and light-heavy quarks.

Fig. 1 shows the pion (light-light) correlation function $G_{\pi}(x)$ and its derivative with respect to $\mu$ at $\beta=5.20$. We perform single pole fit for the data, which turned out to be sufficient for the pion channel.

Fig. 2 shows $\partial m / \partial \mu$ as a function of $T / T_{c}$. Despite the large errors we observe a systematic tendency towards raising the derivative of $\mathrm{m}$ above $T_{c}$.

Fig. 3 shows the response of the coupling $A$, $\partial \ln A / \partial \mu$ as a function of $T / T_{c}$. Both light-light and heavy-light mesons show similar values and no apreciable temperature dependence.

\section{Discussions}

Our preliminary results show remarkable characteristics of the response of meson masses to chemical potential. Possible interpretations for $\partial m / \partial \mu$ of the light-light system are as follows.

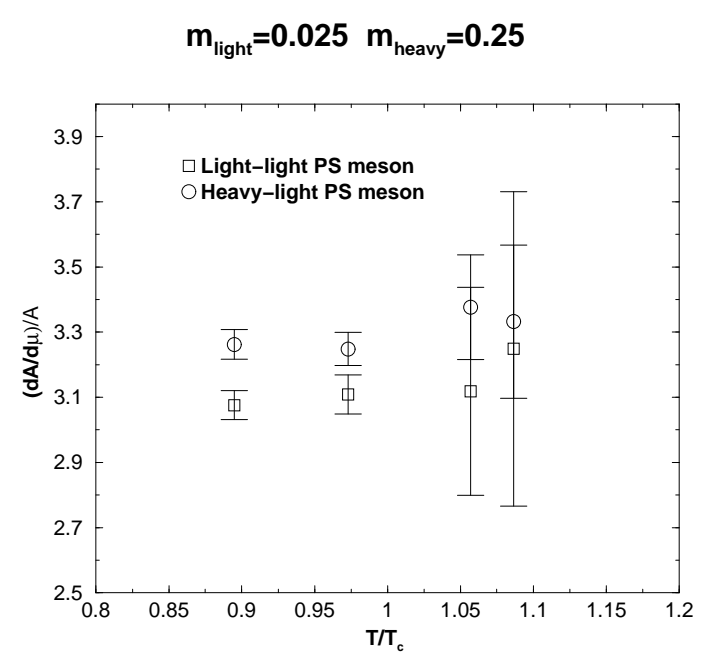

Figure 3. Response of the coupling A to chemical potential, $\partial \ln A / \partial \mu$ as a function of $T / T_{c}$.

The weak response of the mass below $T_{c}$ indicates a persistence ofthe Nambu-Goldstone boson nature at least up to $T=0.97 T_{c}$. Growth of it above $T_{c}$ is consistent with chiral restoration since the meson looses the Nambu-Goldstone character.

Calculations reported here were done on HSP ( NEC ) at INSAM, Hiroshima Univ. This work is supported by the Grant-in-Aid for Scientific Research by Monbusho, Japan (No.11740159).

\section{REFERENCES}

1. T.Hatsuda and S-H.Lee, Phys. Rev. C46 (1992) 34.

2. S. Gottlieb, W. Liu, R. L. Renken, R. L. Sugar, and D. Toussaint, Phys. Rev. D38 (1988) 2888.

3. S. Gottlieb, W. Liu, R. L. Renken, R. L. Sugar, and D. Toussaint, Phys. Rev. D35 (1988) 3972. 